\title{
STABILITY OF HIGH-SPEED BOUNDARY LAYER ON A SHARP CONE WITH LOCALIZED WALL HEATING OR COOLING
}

\author{
V. G. Soudakov ${ }^{1,2}$, A. V. Fedorov ${ }^{1,2}$, and I. V. Egorov ${ }^{1,2}$ \\ ${ }^{1}$ Moscow Institute of Physics and Technology \\ 16 Gagarina Str., Zhukovsky, Moscow Region 140180, Russia \\ ${ }^{2}$ Central Aerohydrodynamic Institute (TsAGI) \\ 1 Zhukovsky Str., Zhukovsky, Moscow Region 140180, Russia
}

\begin{abstract}
A localized heating or cooling effect on stability and transition of the boundary layer flow on a sharp cone is analyzed at the Mach number 6 . The mean flows are calculated using axisymmetric Navier-Stokes equations. The spatial linear stability analysis is performed for twodimensional (2D) disturbances related to the Mack second mode. The transition onset points are estimated using the $e^{N}$ method. In this framework, the heater or cooler may cause earlier or later transition depending on the choice of critical $N$-factor. Direct numerical simulations (DNS) of $2 \mathrm{D}$ wave propagating in the boundary layer are compared with stability results.
\end{abstract}

\section{INTRODUCTION}

Laminar-turbulent transition causes significant increase in heat transfer and viscous drag that leads to severe restrictions on performance of high-speed vehicles. The thermal protection systems (TPS) of such vehicles may have elements of different heat conductivity and/or emissivity. Junctures between these elements lead to jumps of the heat-transfer boundary conditions. These thermal nonuniformities may significantly affect the boundary-layer mean flow, excitation and evolution of unstable modes, and, ultimately, the transition locus. Investigations of physical mechanisms associated with the foregoing thermal effects may help to design new TPS with laminar flow control capabilities.

The wall temperature jump effect was investigated theoretically $[1,2]$ and numerically [3] for laminar supersonic mean flows. Two-dimensional DNS of receptivity and stability of the boundary layer on a flat plate in the Mach 6 free stream were carried out in [4] with emphasis on the case where there is a wall

This is an Open Access article distributed under the terms of the Creative Commons Attribution License 4.0, which permits unrestricted use, distribution, and reproduction in any medium, provided the original work is properly cited. 
temperature jump. Steady-state numerical solutions showed that the positive jump (from cold to hot wall) induces compression waves and leads to thickening of the boundary layer, while the negative jump induces expansion waves and leads to thinning of the boundary layer. The wall temperature jumps were located at different distances from the plate leading edge. These jumps affect both stability and receptivity of the boundary layer. It was found that the total growth of an unstable wave of fixed frequency strongly depends on the sign and locus of the jump.

Localized surface heating or cooling can be considered as a technique for laminar flow control. This method was used to suppress the first mode disturbances (Tollmien-Schlichting waves) $[5,6]$ by local heating in the subsonic boundary layer. A heating strip can be also used for stabilization of the Mack first mode at supersonic speeds, as it was shown in [7] for the boundary layer over a flat plate in the free-stream Mach number 3.5.

The foregoing studies for subsonic and supersonic flows revealed the following mechanism [8]. In the relaxation region developed downstream of the heating strip, the boundary layer temperature is larger than the wall temperature so that the boundary layer "sees" a relatively cold wall. According to the linear stability theory, this leads to decreasing of the first mode growth rates.

The influence of a nonuniform cooling on the Mack second mode in the boundary layer on flat plates and sharp cones was investigated in [9] at the free-stream Mach number of 6.84. Cooling with a ramp decrease in the wall temperature was considered. The boundary layer equations were used for calculations of the boundary-layer mean flow. However, these equations are not applicable to the regions where the temperature boundary conditions have sudden changes.

In the present work, a localized heating or cooling is considered on a sharp cone at zero angle of attack at the free-stream Mach number 6. The steadystate laminar flow solution is calculated using the Navier-Stokes equations. This solution provides the mean flow for further predictions of the growth rates and amplifications of unstable disturbances using the linear stability theory (LST). The stability data are used to estimate the transition onset points with the help of the $e^{N}$ method. The LST results are compared with 2D DNS of 2D unstable wave propagating in the boundary layer.

\section{PROBLEM FORMULATION}

Viscous axisymmetric unsteady compressible flows are described by the NavierStokes equations. Numerical simulations are carried out for a sharp cone of 7 degree half angle. The flow variables are made nondimensional using the freestream parameters denoted by the subscript " $\infty: " u=u^{*} / U_{\infty}^{*}$ and $v=v^{*} / U_{\infty}^{*}$ are the longitudinal and vertical velocity components; $p=p^{*} /\left(\rho_{\infty}^{*} U_{\infty}^{* 2}\right)$ is the 
pressure; $\rho=\rho^{*} / \rho_{\infty}^{*}$ is the density; and $T=T^{*} / T_{\infty}^{*}$ is the temperature. The nondimensional coordinates are $x=x^{*} / L^{*}$ and $y=y^{*} / L^{*}$ and time is $t=t^{*} U_{\infty}^{*} / L^{*}$ where $U_{\infty}^{*}$ is the free-stream velocity; $L^{*}$ is the cone length; and $x$ is measured from the cone tip along the cone axis. Hereinafter, asterisks denote dimensional variables. The fluid is a perfect gas with the specific heat ratio $\gamma=1.4$ and Prandtl number $\operatorname{Pr}=0.72$. Calculations are performed for the free-stream Mach number 6 and different Reynolds numbers (based on the freestream parameters and the cone length) $\operatorname{Re}_{L}=\rho_{\infty}^{*} U_{\infty}^{*} L^{*} / \mu_{\infty}^{*}$ where $\mu_{\infty}^{*}$ is the free-stream dynamic viscosity. The viscosity-temperature dependence is approximated by the Sutherland law with the Sutherland constant $110.4 \mathrm{~K}$. The flow parameters correspond to the experimental conditions of the Transit-M wind tunnel of Institute of Theoretical and Applied Mechanics (ITAM, Novosibirsk) for the cone model of length $L^{*}=0.5 \mathrm{~m}$.

Details on the problem formulation and the governing equations are given in [10]. The computational domain is a rectangle with its bottom side corresponding to the cone surface for the region $0 \leq x \leq 1$. The boundary conditions on the cone surface are the no-slip condition and the constant wall temperature $T_{w \text { base }}^{*}=292 \mathrm{~K}$. On the outflow boundary, the unknown dependent variables are extrapolated using the linear approximation. On the inflow and upper boundaries, the conditions correspond to the undisturbed free stream. The upper boundary is located above the cone-induced shock wave.

To simulate heating/cooling source, the wall temperature is specified as

$$
T_{w}= \begin{cases}T_{w \text { base }}^{*}+\frac{\Delta T_{h}}{2} \tanh \left(70\left(x-x_{S 1}\right)\right)+\frac{\Delta T_{h}}{2}, & x \in\left[0.1, \frac{x_{S 1}+x_{S 2}}{2}\right] ; \\ T_{w \text { base }}^{*}-\frac{\Delta T_{h}}{2} \tanh \left(70\left(x-x_{S 2}\right)\right)+\frac{\Delta T_{h}}{2}, & x \in\left[\frac{x_{S 1}+x_{S 2}}{2}, 0.5\right] \\ T_{w \text { base }}^{*}, & x \notin[0.1,0.5]\end{cases}
$$

where $x_{S 1}=0.2524\left(x_{S 1}^{*}=126.2 \mathrm{~mm}\right)$ is the upstream boundary of the heater/ cooler and $x_{S 2}=0.4072\left(x_{S 2}^{*}=203.6 \mathrm{~mm}\right)$ is its downstream boundary. The temperature distribution is smoothed near the boundaries in accord with the experimental measurements of $T_{w}^{*}\left(x^{*}\right)$.

The problem is solved numerically using the implicit second-order finitevolume method described in [10]. The 2D (axisymmetric) Navier-Stokes equations are approximated by a shock-capturing scheme that allows for modeling of flow nonuniformities in the temperature jump vicinity. The advection terms are approximated by the third-order WENO (weighted essentially nonoscillatory) scheme to decrease the numerical dissipation. Herein, the computational grid has $478 \times 599$ nodes. The grid is clustered in the direction normal to the cone surface so that the boundary-layer region contains approximately $50 \%$ of nodes. The code algorithm as well as its implementations and validations are discussed in $[10]$. 


\section{STEADY-STATE SOLUTION WITH AND WITHOUT HEATING/COOLING}

First, the steady-state solution is calculated to provide the mean flow field for the baseline case without heating or cooling. The pressure field is shown in Fig. 1. Using this numerical solution, the flow parameters on the upper boundary-layer edge (denoted by subscript " $e$ ") can be determined. In the mid station $x=0.5$, these parameters are $T_{e}=1.225, U_{e}=0.984, \mathrm{M}_{e}=5.33$, and the unit Reynolds number $\operatorname{Re}_{e 1} \approx 11 \cdot 10^{6} \mathrm{~m}^{-1}$.

For the case of heating (or cooling), the wall temperature rise (or drop) on the heated (or cooled) surface strip is $\Delta T_{h}$. Five different temperature distributions were numerically simulated: Run 1403 - baseline flow without heating or cooling; Run 1419 - strong cooling; Run 1421 — weak cooling; Run 1458 weak heating; and Run 1446 - strong heating. These runs correspond to experimental conditions of the ITAM Transit-M wind tunnel. The free-stream and surface parameters are given in Table 1 . The corresponding wall-temperature distributions, which are calculated using (1), are shown in Fig. $2 a$. It should be noted that the temperature difference $\left|\Delta T_{h}\right|$ for the case of strong heating is approximately the same as in the case of weak cooling.
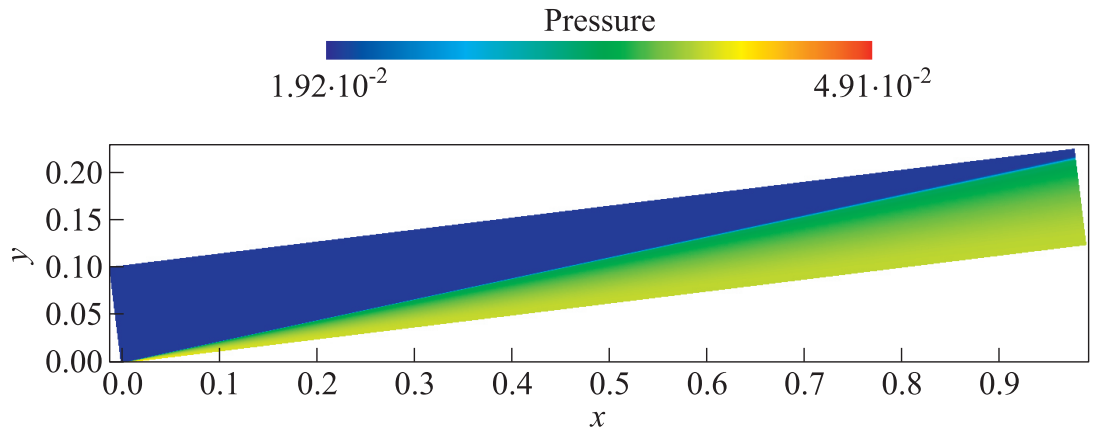

Figure 1 The mean-flow pressure field past the cone without heating or cooling

Table 1 Free-stream and surface parameters

\begin{tabular}{lcccccc}
\hline \multicolumn{1}{c}{ Name } & $\mathrm{Run}$ & $\mathrm{M}_{\infty}$ & $\mathrm{Re}_{\infty L}, \mathrm{mio}$ & $T_{\infty}^{*}, \mathrm{~K}$ & $T_{w}^{*}, \mathrm{~K}$ & $\Delta T_{h}^{*}, \mathrm{~K}$ \\
\hline Baseline & 1403 & 6 & 4.51 & 44.24 & 292 & 0 \\
Strong cooling & 1419 & 6 & 4.45 & 44.94 & 292 & -204.6 \\
Weak cooling & 1421 & 6 & 4.44 & 44.61 & 292 & -146.2 \\
Weak heating & 1458 & 6 & 4.57 & 43.85 & 292 & 85.7 \\
Strong heating & 1446 & 6 & 4.46 & 44.70 & 292 & 151.7 \\
\hline
\end{tabular}




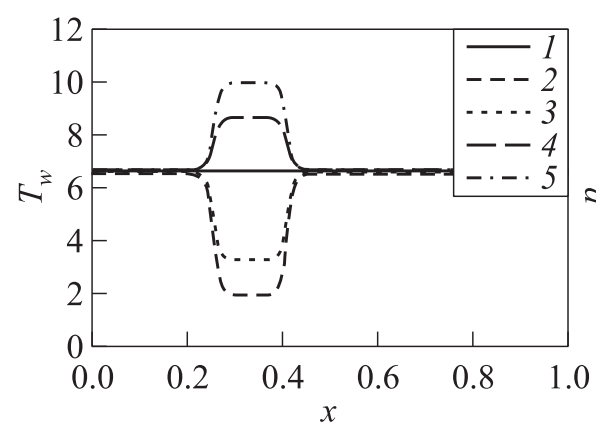

(a)

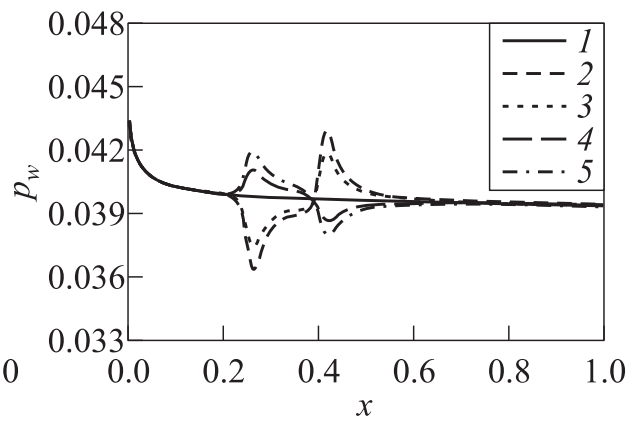

(b)

Figure 2 The wall temperature distributions $T_{w}(x)(a)$ and the wall pressure distribution $p_{w}(x)(b)$ for the cases given in Table 1: 1 - no source; 2 - strong cooling; 3 - weak cooling; 4 - weak heating; and 5 - strong heating

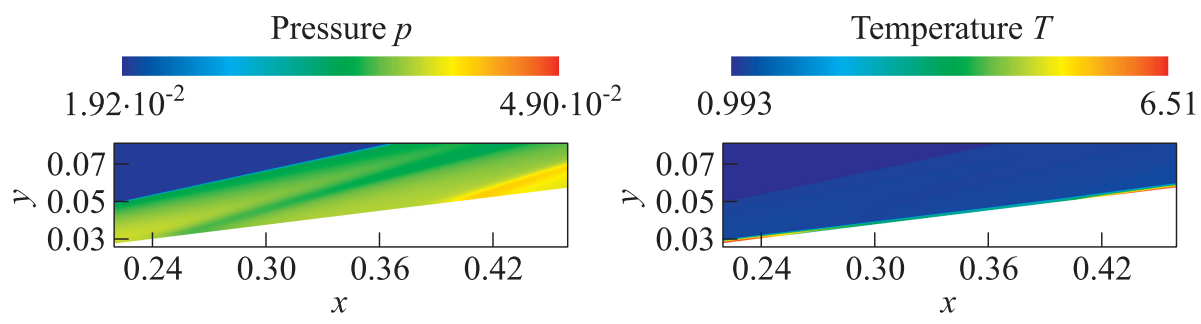

(a)

Temperature $T$
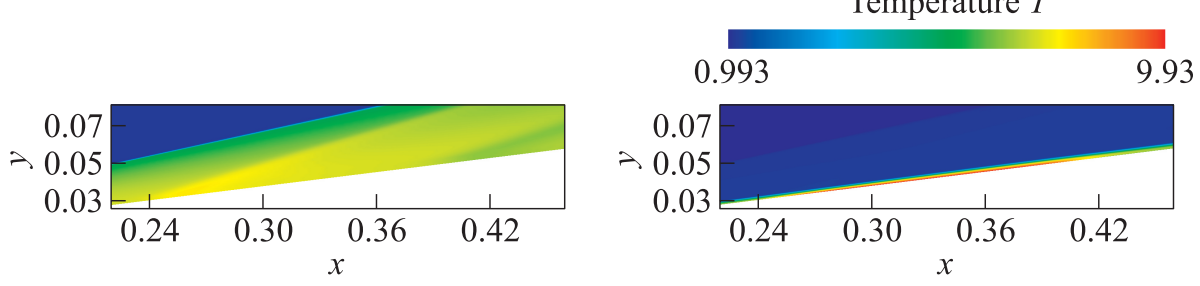

(b)

Figure 3 Pressure (left column) and temperature (right column) fields of the mean flow near the cone surface with the strong cooling strip (Run 1419) (a) and strong heating strip (Run 1446) (b); $0.22 \leq x \leq 0.46$

Figure $3 a$ shows the pressure and temperature fields in the range of 0.22 $\leq x \leq 0.46$ for the strong cooling case. Because of the wall temperature drop near the upstream boundary $x_{S 1}=0.2524$, the boundary-layer temperature and its thickness decrease downstream. This causes expansion waves emanating from the drop vicinity. Near the downstream boundary $x_{S 2}=0.4072$, the wall temperature rise produces an opposite effect causing compression waves. 


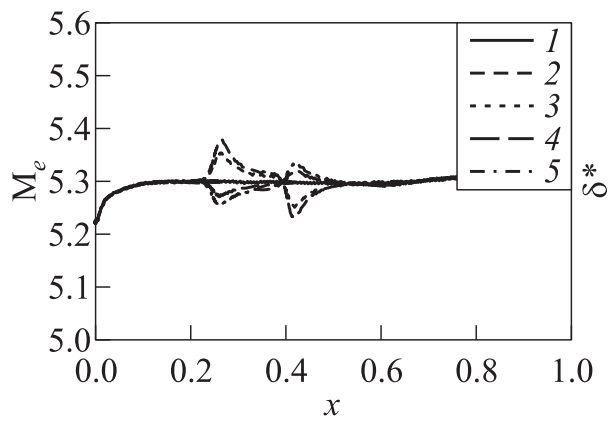

(a)

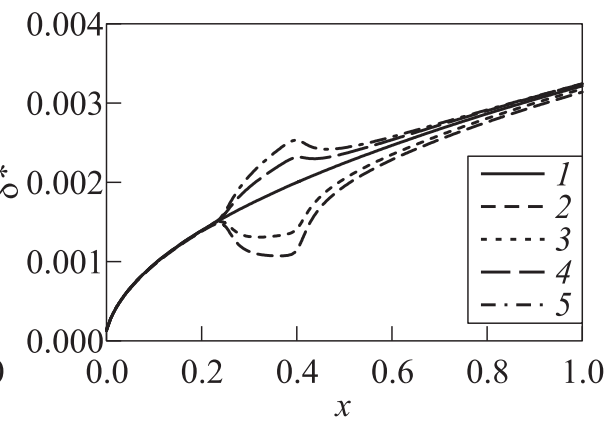

(b)

Figure 4 The Mach number distributions along the upper boundary-layer edge $(a)$ and the boundary-layer displacement thickness $(b)$ for the cases given in Table 1: $1-$ no source; 2 - strong cooling; 3 - weak cooling; 4 - weak heating ; and 5 - strong heating
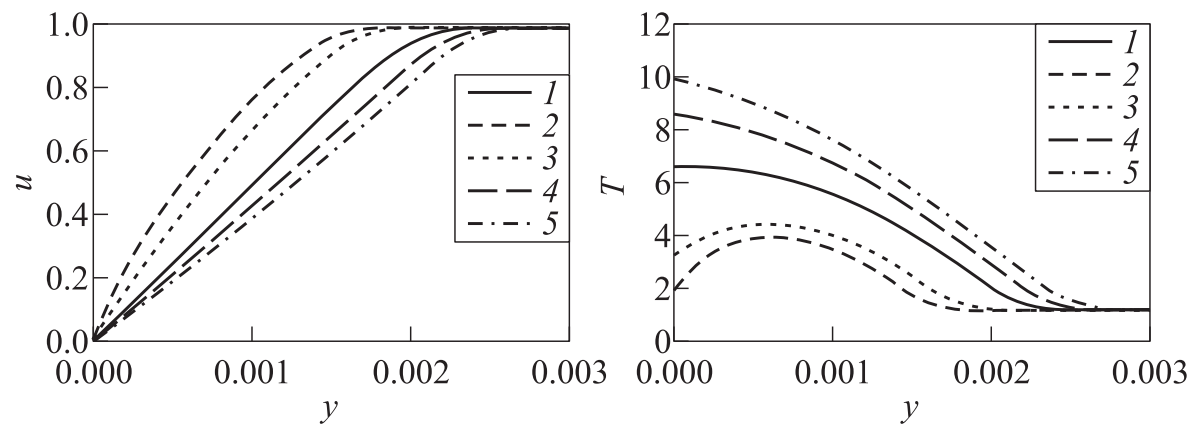

(a)
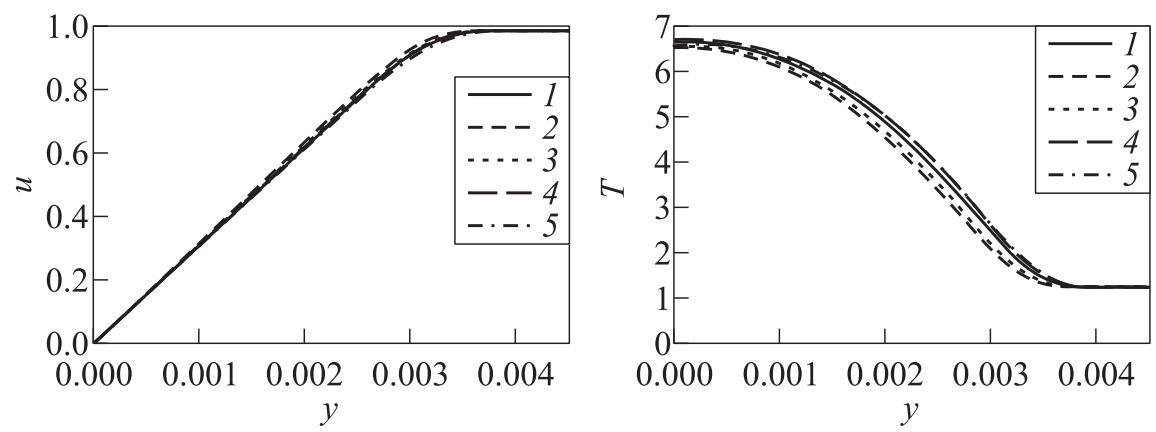

(b)

Figure 5 Velocity and temperature profiles at $x=0.3(a)$ and $0.75(b): 1-$ no source; 2 - strong cooling; 3 - weak cooling; 4 - weak heating; and 5 - strong heating 
The same fields are shown in Fig. $3 b$ for the heating strip. Now, the wall temperature rises near the upstream boundary $x=x_{S 1}$ and drops near the downstream boundary $x=x_{S 2}$. The compression waves are formed near the first point, while the expansion waves are formed near the second point.

The wall temperature and pressure distributions for the all cases are shown in Fig. 2. The Mach number distributions $\mathrm{M}_{e}(x)$ along the upper boundarylayer edge are shown in Fig. $4 a$. The displacement thickness distributions $\delta^{*}$ are shown in Fig. $4 b$. It is seen that there is a small upstream influence of the heating/cooling strip on the mean flow (see, e.g., Fig. 2b). The downstream influence protrudes to approximately 1-2 strip lengths (see Figs. $2 b$ and 4 ). In the case of strong cooling, the minimal displacement thickness is approximately one half of the undisturbed $\delta^{*}$.

The velocity and temperature vs. the wall normal coordinate $y$ are shown in Fig. $5 a$ for the station $x=0.30$ located near the strip center. For the cold strips (curves 2 and 3 ), the velocity profiles are more filled than in the baseline case (curves 1). For the hot strips (curves 4 and 5), the trend is opposite. Similar velocity and temperature profiles are presented in Fig. $5 b$ for the station $x=0.75$ located in the relaxation region (downstream of the heating/cooling region). As expected, they are close to the baseline profiles. As will be shown in the next section, the mean-flow altering induced by the cooler/heater strongly affects the growth rates of unstable waves.

\section{LINEAR STABILITY ANALYSIS}

The growth rates and downstream amplifications of convectively unstable disturbances are computed with the help of the in-house linear stability code. This code solves the linear stability equations for compressible boundary-layer flow using a 4th-order Runge-Kutta scheme and a Gram-Schmidt orthonormalization procedure. The eigenvalues of the discrete spectrum are calculated with the help of a shooting/Newton-Raphson procedure. Hereafter, the authors focused on the Mack second mode whose maximal growth rates correspond to $2 \mathrm{D}$ waves. In accord with the local-parallel LST, the disturbance is expressed in the wave form:

$$
q=\hat{q}(y) \exp [(\alpha x-\omega t)]
$$

where $\omega$ is the real circular frequency and $\alpha(\omega)=\alpha_{r}+i \alpha_{i}$ is the complex eigenvalue. The downstream growth of instability is characterized by the $N$-factor:

$$
N(x, \omega)=\int_{x_{0}}^{x} \sigma(x, \omega) d x
$$

where $\sigma=-\alpha_{i}$ is the spatial growth rate and the initial point $x_{0}$ should be determined from the receptivity problem. In the standard $e^{N}$ method [11], it is assumed that all unstable waves are equally available allover the body surface and 
the initial points lie on the lower neutral branch, $x_{0}=x_{n}(\omega)$. The $N$-factors are computed at various fixed dimensional frequencies $\omega^{*}$. The transition onset point $x_{\mathrm{tr}}$ is estimated from the equation $\max _{\omega^{*}} N\left(x_{\mathrm{tr}}, \omega^{*}\right)=N_{\mathrm{tr}}$ where $N_{\mathrm{tr}}$ is the empirical constant. Note that the stability calculations agree well with the results of STABL code [12], where the boundary-layer flow past a sharp 7 degree half-angle cone at zero angle of attack was considered at the free-stream Mach number 10.

First, let consider the characteristics of the most unstable waves. Figure 6 shows the distributions of maximal growth rates $\sigma_{\max }(x)=\max _{\omega}[\sigma(x, \omega)]$ for the cases given in Table 1. Here, the growth rates are scaled using the Blasius length $\sqrt{x^{*} u_{e}^{*} / U_{e}^{*}}$. The instability is affected by the wall-temperature difference and the mean-flow pressure gradient. As shown in Fig. $2 b$, the cooling induces a strong favorable pressure gradient near the upstream boundary of the strip $x=x_{S 1}$

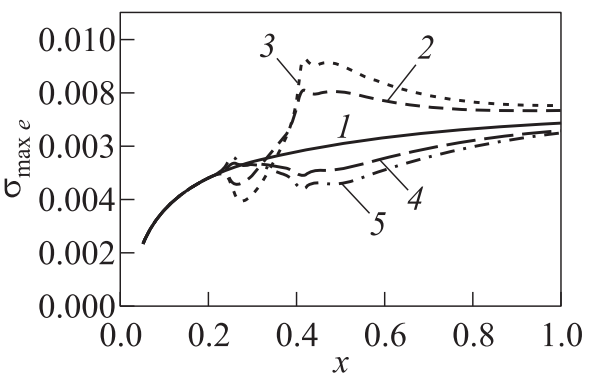

Figure 6 Maximal growth rates for the cases given in Table 1: 1 - no heat; 2 weak cooling, smooth; 3 - strong cooling, smooth; 4 - weak heating, smooth; and 5 strong heating, smooth that causes a sharp drop of $\sigma_{\max }$. Further downstream, the mean-flow pressure recovers and $\sigma_{\max }$ increases in the cooling region. Then, a sharp peak of $p_{w}(x)$ near the downstream boundary $x=x_{S 2}$ causes a positive peak of $\sigma_{\max }$. Downstream of the cooling region, the growth rate is higher than in the baseline case and it relaxes slowly to the baseline distribution. The heating produces an opposite effect on the wall pressure distribution that leads to the positive peak of $\sigma_{\max }$ near the upstream boundary $x=x_{S 1}$ and the negative peak near the downstream boundary $x=x_{S 2}$. Downstream of the heater, the growth rate is lower than in the baseline case and it relaxes slowly to the baseline distribution. Because the cooling/heating effects on the wall pressure distribution (see Fig. $2 b$ ) and the $\sigma_{\max }$ distribution (see Fig. 6) are qualitatively similar, it is reasonable to assume that the second-mode instability is predominantly controlled by the induced pressure gradient. The wall-temperature ratio seems to be less effective.

Note that jumps of the wall temperature near the cooling/heating boundaries cause local nonuniformities of the mean flow where the nonparallel effects seem to be strong. Since the local-parallel stability analysis near these boundaries is not valid, the $e^{N}$ results presented hereafter should be treated as a first-cut estimate of the cooling/heating effect.

Figure 7 shows $N$-factors at various frequencies where $N(x)$ are computed from the initial points $x_{0}=x_{n}\left(\omega^{*}\right)$. Upstream of the cooling strip, the $N$-factors 

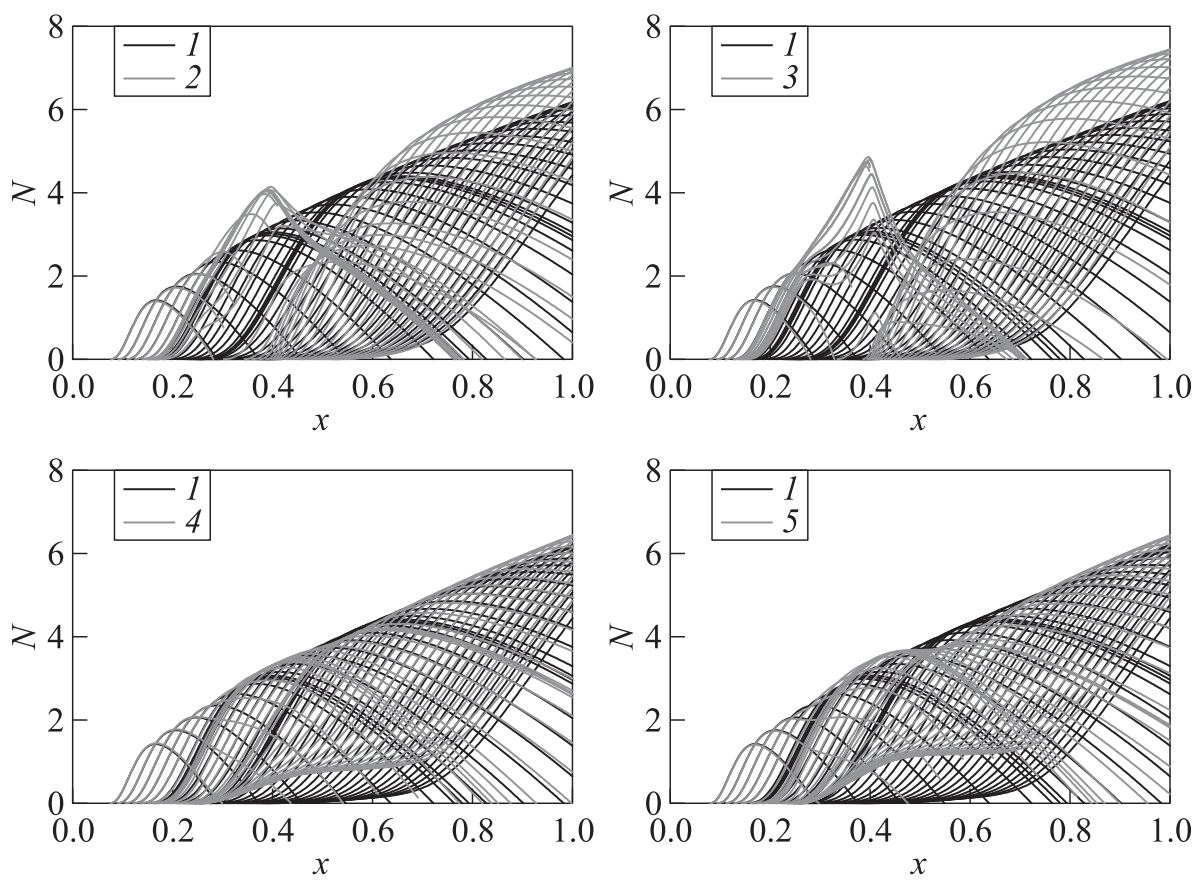

Figure $7 N$-factors for the wall heating/cooling cases: 1 - no heat, Run 1403 (baseline case); 2 — weak cooling, Run 1421; 3 — strong cooling, Run 1419; 4 — weak heating, Run 1458; and 5 - strong heating, Run 1446

are not affected. In the cooling region (upper plots), the $N$-factor envelope is higher than in the baseline case. Then, it decreases sharply and rises again overshooting the baseline envelope in the downstream relaxation region. In the strong-cooling case, the stabilization effect near the downstream boundary of the strip is so strong that the growth rates drop to high negative values and some of lines $N(x)$ are terminated. Distributions of $N$-factors for the weak (Run 1458) and strong (Run 1446) heating cases are shown in the lower plots. As in the cooling cases, the heating does not affect the second-mode instability in the upstream region $x<x_{S 1}$. Although the heating reduces the maximal growth rates for $x>x_{S 2}$, the unstable region of low-frequency waves is broadened. Therefore, the $N$-factor envelope is affected weakly.

\section{DIRECT NUMERICAL SIMULATION}

Direct numerical simulations are performed for the baseline case (Run 1403), the strong cooling case (Run 1419), and the strong heating case (Run 1446). First, 


\section{Pressure}

$-4 \cdot 10^{-5} 4 \cdot 10^{-5}$

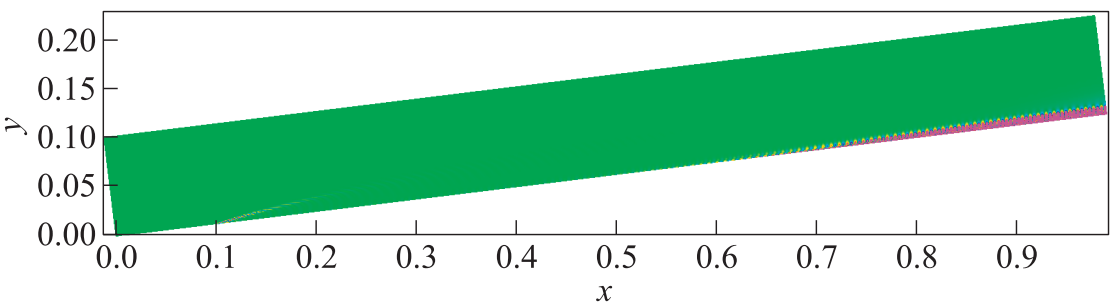

Figure 8 Pressure disturbance field for the baseline case
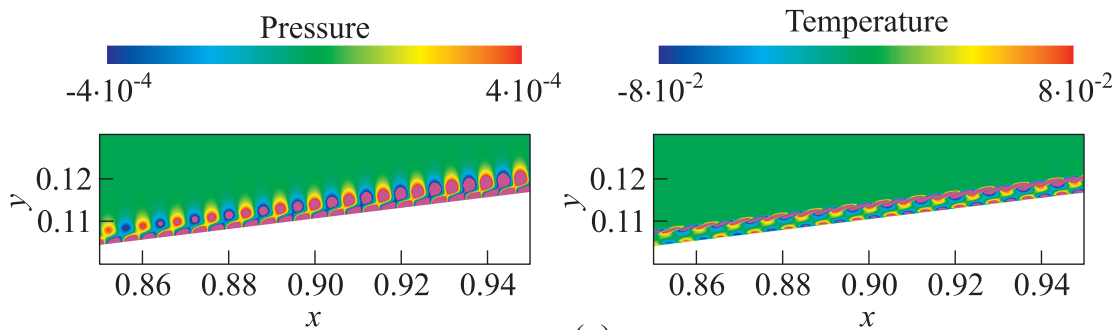

(a)
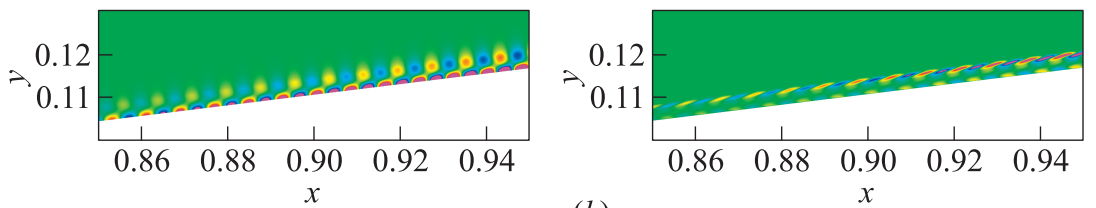

(b)
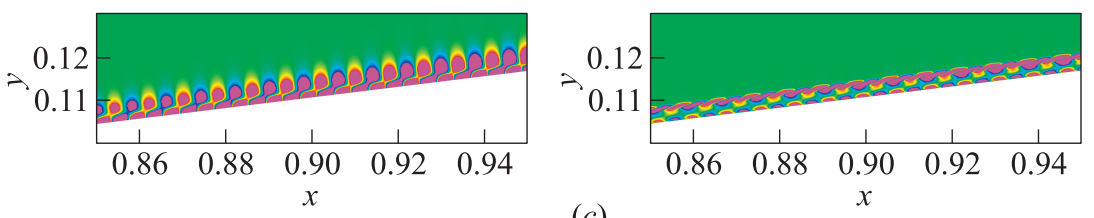

(c)

Figure 9 Pressure (left column) and temperature (right column) disturbance fields, $0.85 \leq x \leq 0.95$ : $(a)$ baseline; $(b)$ strong cooling; and $(c)$ strong heating

a steady-state solution, which satisfies the free-stream boundary conditions on the inflow and upper boundaries, is calculated to provide the mean flow on fine mesh. The computational grid has $6397 \times 449$ nodes. Then, time-harmonic disturbances are induced by a local periodic suction-blowing with the mass flow rate on the cone surface: 


$$
q_{w}(x, t)=\frac{\rho_{w}^{*} v_{w}^{*}}{\rho_{\infty}^{*} v_{\infty}^{*}}=\varepsilon \sin \left(2 \pi \frac{x-x_{1}}{x_{2}-x_{1}}\right) \sin (\omega t), \quad x_{1} \leq x \leq x_{2}, \quad t>0,
$$

where $\varepsilon$ is the forcing amplitude; and $x_{1}=0.1$ and $x_{2}=0.114$ are the boundaries of the suction-blowing region. The circular frequency is $\omega=\omega^{*} L^{*} / U_{\infty}^{*}=700$. The suction-blowing amplitude, $\varepsilon=10^{-4}$, was chosen small enough to compare numerical results with LST. In the unsteady problem, the temperature disturbances on the wall are zero.

The local blowing-suction excites unstable disturbances in the boundary layer reaching its maximum near the end of computational domain. The pressure disturbance field which is the difference between an instantaneous field and the mean-flow field is shown in Fig. 8 for Run 1403 (the baseline case). For $x>0.6$, the downstream growing disturbance is observed in the boundary layer.

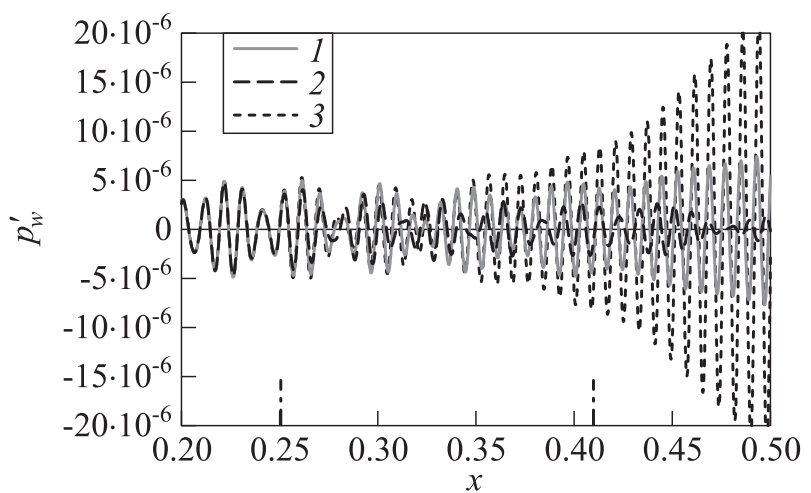

(a)

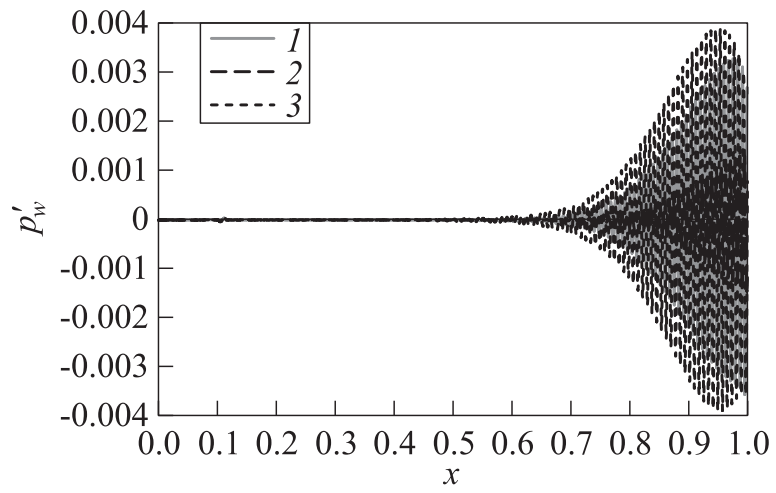

(b)

Figure 10 Wall-pressure disturbances near the cooling/heating strip, $0.2 \leq x$ $\leq 0.5(a)$ and global distributions $(b), \omega=700: 1$ - baseline; 2 - strong cooling; and 3 - strong heating 
Detailed view of this instability in the region $0.85 \leq x \leq 0.95$ is shown in Fig. 9 for the baseline case and the strong heating/cooling cases. The pressure disturbances have a two-cell structure in vertical direction, whereas the temperature disturbances have rope-like structure near the upper boundary-layer edge. These patterns are typical for the Mack second mode [10]. Maximal amplitudes are observed in the strong-heating case, while the minimal amplitudes correspond to the strong-cooling case.

Figure $10 a$ shows the details of the wall pressure distributions $p_{w}^{\prime}(x)$ in the vicinity of heating/cooling strip. The strip boundaries $x_{S 1}$ and $x_{S 2}$ are marked by the vertical dashdotted lines. The amplitude modulations are, presumably, due to the Mack first mode of relatively large wavelength. In the upstream region $x<x_{S 1}$, there is no noticeable difference between $p_{w}^{\prime}(x)$ for the all three cases. In the strip region $x_{S 1}<x<x_{S 2}$, the disturbance amplitude decreases on the cold strip, while it increases on the hot strip. Further downstream $\left(x>x_{S 2}\right)$, the disturbance starts to grow with appreciable rate in the heating case and with weaker rate in the baseline case. For the cooling case, the disturbance amplitude decreases weakly. Note that the disturbances evolve smoothly through the cooling/heating region. The disturbance field patterns (not shown here) do not contain acoustic waves or other additional disturbances, which could be induced by scattering of the primary wave on the strip boundaries.

The global distributions of $p_{w}^{\prime}$ are shown in Fig. 10b. The maximal wallpressure disturbances are reached in the case of strong heating, while the minimal - in the case of strong cooling. This trend is opposite to those shown in Fig. 7.

\section{COMPARISON OF DIRECT NUMERICAL SIMULATION AND LINEAR STABILITY THEORY RESULTS}

To clarify the aforementioned difference, additional LST computations were performed for the second-mode wave with the frequency being equal to the suctionblowing frequency of DNS $(\omega=700)$. The LST distributions of dimensional growth rates $\sigma^{*}(x)$ and the amplitude ratio $A(x)=\exp (N(x, \omega))$ are shown in Fig. $11 a$ where the initial point is the neutral point, $x_{0}=x_{n}(\omega)$. Similar to DNS solutions, the heating leads to higher disturbances (compare curves 1 and 3 ). However, the cooling causes even stronger destabilization that is contrasted with the DNS solution showing stabilization effect (see Fig. 10b).

Because the DNS solutions were obtained using a 2D blowing-suction which generates disturbances near the point $x_{S 1}=0.1$, the second-mode wave is excited at this point and propagates downstream. The cooling/heating element does not affect the mean flow in the upstream region where the blowing-suction is located. 

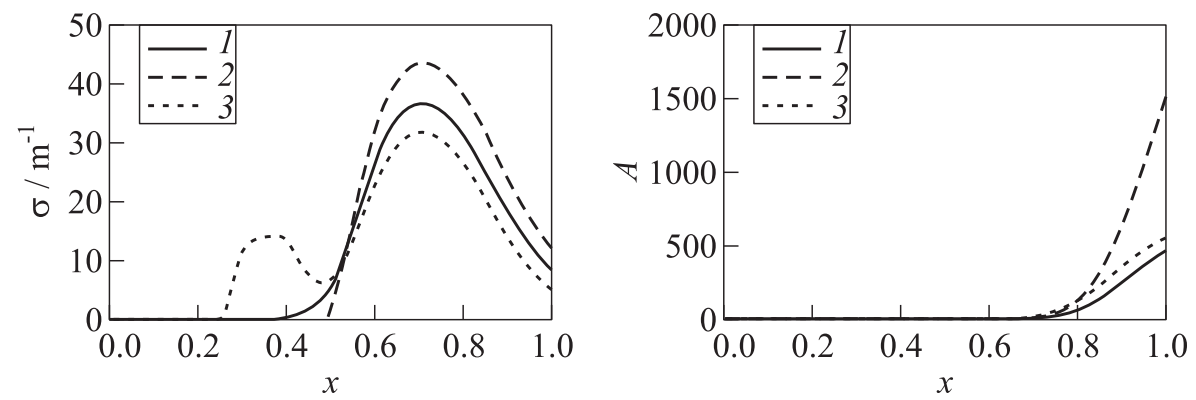

(a)
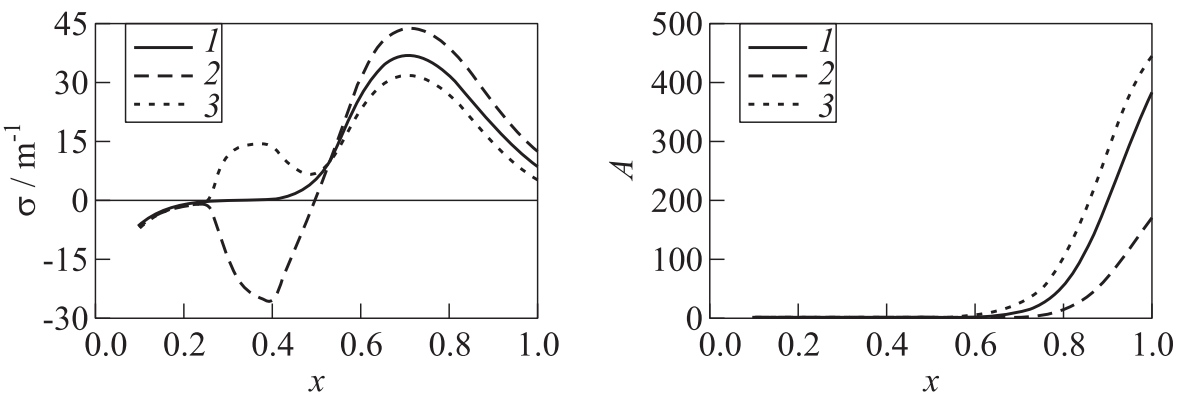

(b)

Figure 11 Growth rates and the amplitude ratio of the second mode at the DNS frequency $\omega=700$, LST computations from the neutral point $(a)$ and from $x_{0}=0.1(b)$ : 1 - no heat; 2 - strong cooling; and 3 - strong heating

The free-stream parameters are almost the same for the all three cases. These arguments allow to assume that the initial amplitudes of unstable wave are the same at the $x_{0}=x_{S 1}$ in the all three cases. With this assumption, let compute the growth rates and the amplification ratios starting from the point $x_{0}=0.1$ (Fig. 11b). The damping of unstable mode in the upstream region $x_{0}<x<x_{n}$ is relatively weak and it is about the same in the cases of no-heat and strong heating (curves 1 and 3). For the strong cooling case (curves 2), the

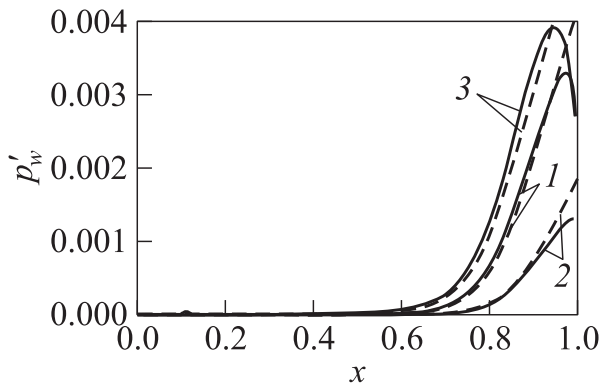

Figure 12 Comparisons of LST predictions (dashed curves) with the DNS solutions (solid curves) of amplitudes of wall pressure disturbances, $\omega=700$ : 1 - baseline; 2 - strong cooling; and 3 - strong heating. Computations from $x_{0}=0.1$ 


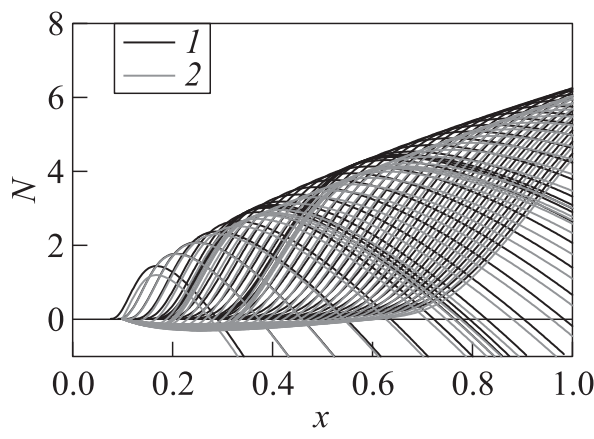

(a)

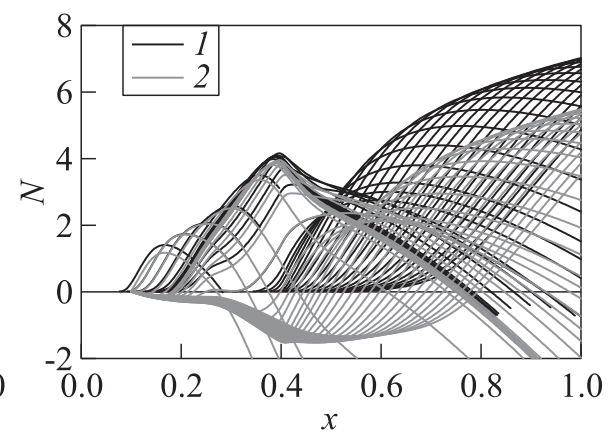

(b)

Figure $13 N$-factors computed from the neutral points (1) and from the fixed point $x_{0}=0.1(2)$ for the baseline case $(a)$ and the weak cooling case $(b)$

damping is strong that leads to significant reduction of the amplification ratio. Figure 12 shows comparisons of LST predictions with the DNS solutions. Since the LST analysis does not account for receptivity, the initial amplitudes of LST solutions are arbitrary. In this figure, the amplitude ratio is scaled by a factor providing the best fit for the baseline case (curves 1 ). The same factor is used for scaling in the two other cases. The agreement between DNS and LST solutions is satisfactory accounting for the fact that the LST analysis ignores the nonparallel effects, which are expected to be strong in the heating/cooling region.

This example indicates that the cooling effect essentially depends on the initial point from which the unstable waves start to propagate downstream. This point, in turn, is determined by the receptivity mechanism. To illustrate this

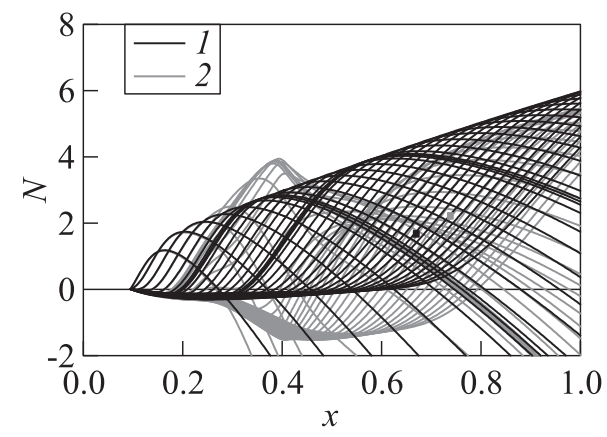

Figure $14 N$-factors computed from the fixed point $x_{0}=0.1$ for the baseline case (1) and the weak cooling case (2), markers denote the lines corresponding to $\omega=700$ statement, let consider the following situation. Assume that the second-mode waves of all frequencies are effectively excited near the cone tip and the receptivity process is completed by the station $x_{0}=0.1$. It is also assumed that in this station, the initial amplitudes of unstable waves are approximately the same for all frequencies. In this case, the $N$-factors and their envelopes should be calculated from the fixed initial point $x_{0}=0.1$. Figure 13 compares these $N$-factors (curves 2) with $N$-factors computed from the neutral points (curves 1) 
for the cases of no-heating (Fig. 13a) and weak cooling (Fig. 13b). In the baseline case, the damping of instability in the upstream regions $x_{0}<x<x_{n}$ is relatively weak and the $N$-factor envelopes are close to each other. In the cooling case, the situation is different. The low-frequency waves, which are dominant downstream from the strip, experience a strong damping in the regions $x_{0}<x<x_{n}$, and the $N$-factor envelope (curves 2) is shifted down significantly at $x>0.6$. As shown in Fig. 14, stability computations at the fixed initial point $x_{0}=0.1$ predict that the cold strip produces a stabilization effect for $x>0.6$, just opposite to the case of $x_{0}=x_{n}$ shown in Fig. 7 .

\section{SUMMARY}

A localized heating or cooling effect on stability of the boundary-layer flow on a sharp cone at zero angle of attack is analyzed at the free-stream Mach number 6 . Five different temperature distributions were numerically simulated: baseline flow without heating or cooling, strong and weak cooling, and strong and weak heating. The free-stream and surface conditions correspond to the experiments recently performed in the ITAM Transit-M wind tunnel (Novosibirsk).

The mean flows are calculated using axisymmetric Navier-Stokes equations. The spatial LST analysis is performed for 2D disturbances related to the Mack second mode. The $N$-factors computed from the neutral points indicate that the cooling strip may cause earlier transition, while the heating strip produces a weak effect.

To account for the nonparallel effects, which are enhanced by the cooling/heating strip, DNS was performed for 2D disturbance excited by the suctionblowing slot located upstream from the strip. It was shown that the second mode instability is a dominant component of the disturbance field in the boundary layer downstream of the strip. It was found that the hot strip leads to increasing of the instability amplitude, while the cold strip produces an opposite effect. The LST computations of the second-mode amplification starting from the suction-blowing locus agree satisfactory with the DNS solutions. Consequently, the nonparallel effects are not dramatic for the unstable waves passing through the cold or hot region.

The LST computations performed from the different initial points showed that predictions of the wall cooling effect essentially depend on the choice of $x_{0}$. If the $N$-factors are computed from the neutral points, the cold strip leads to the upstream shift of the $N$-factor envelope and, thereby, may cause earlier transition. If the $N$-factors are computed form the fixed point located upstream from the strip, the trend is opposite. This indicates that the local cooling effect essentially depends on the receptivity mechanism (more specifically, on the location of most receptive region). This also suggests that the location of the cooling/heating strip is a critical factor. 


\section{ACKNOWLEDGMENTS}

This work was partially supported by TransHyBeriAN Project of the 7th Framework Program and by the Russian Government under grant 'Measures to Attract Leading Scientists to Russian Educational Institutions' (contract No. 11.G34.31.0072).

\section{REFERENCES}

1. Sokolov, L. A. 1975. Toward an asymptotic theory of plane flows of a laminar boundary layer with a temperature discontinuity on the body. Trudy TsAGI 1650:18-23. [In Russian.]

2. Neiland, V. Ya., V. V. Bogolepov, G. N. Dudin, and I. I. Lipatov. 2008. Asymptotic theory of supersonic viscous gas flows. Elsevier. Ch. 3.5.

3. Inger, G. R., and P. A. Gnoffo. 2001. Analytical and computational study of wall temperature jumps in supersonic flow. AIAA J. 39(1):79-87.

4. Soudakov, V. G., I. V. Egorov, and A. V. Fedorov. 2008. Numerical simulation of receptivity of a hypersonic boundary layer over a surface with temperature jump. 6th European Symposium on Aerothermodynamics for Space Vehicles Proceedings. ESA SP-659.

5. Kazakov, A. V., M. N. Kogan, and V.A. Kuparev. 1985. On the enhancement of the stability of a subsonic boundary layer by heating the surface near the leading edge of the body. Dokl. Akad. Nauk SSSR 3:333.

6. Dovgal, A. V., V.Y. Levchenko, and V. A. Timofeev. 1990. Boundary layer control by a local heating of the wall. Laminar-turbulent transition. Eds. D. Arnal and R. Miche. Berlin-Heidelberg - New York: Springer-Verlag. 113-121.

7. Masad, J. A., and R. Abid. 1995. On transition in supersonic and hypersonic boundary layers. Int. J. Eng. Sci. 33(13):1893-1919.

8. Arnal, D., and J. P. Archambaud. 2008. Laminar-turbulent transition control: NLF, LFC, HLFC. Advances in laminar-turbulent transition modeling. RTO-EN-AVT$151-15$.

9. Gasperas, G. 1989. Effect of wall temperature distribution on the stability of the compressible boundary layer. AIAA Paper No. 89-1894.

10. Egorov, I. V., A. V. Fedorov, and V. G. Soudakov. 2006. Direct numerical simulation of disturbances generated by periodic suction-blowing in a hypersonic boundary layer. Theor. Comput. Fluid Dyn. 20(1):41-54.

11. Jaffe, N. A., T. T. Okamura, and A. M. O. Smith. 1970. Determination of spatial amplification factors and their application to predicting transition. AIAA J. 8(2):301308.

12. Fedorov, A.V., and N.D. Malmuth. 2008. Parametric studies of hypersonic laminar flow control using a porous coating of regular microstructure. AIAA Paper No. 2008-588. 